\title{
Véronique Castellotti, Didier de Robillard (ed.), France, pays de contact de langues
}

\section{Ruggero Druetta}

\section{(2) OpenEdition}

1 Journals

\section{Édition électronique}

URL : http://journals.openedition.org/studifrancesi/33956

DOI : 10.4000/studifrancesi.33956

ISSN : 2421-5856

Éditeur

Rosenberg \& Sellier

\section{Édition imprimée}

Date de publication : 1 décembre 2005

Pagination : 697-699

ISSN : 0039-2944

\section{Référence électronique}

Ruggero Druetta, "Véronique Castellotti, Didier de Robillard (ed.), France, pays de contact de langues », Studi Francesi [En ligne], 147 (XLX | III) | 2005, mis en ligne le 30 novembre 2015, consulté le 18 avril 2021. URL : http://journals.openedition.org/studifrancesi/33956 ; DOI : https://doi.org/10.4000/ studifrancesi.33956

Ce document a été généré automatiquement le 18 avril 2021.

\section{(c) 9 (i) $\Theta$}

Studi Francesi è distribuita con Licenza Creative Commons Attribuzione - Non commerciale - Non opere derivate 4.0 Internazionale. 


\title{
Véronique Castellotti, Didier de Robillard (ed.), France, pays de contact de langues
}

\author{
Ruggero Druetta
}

\section{RÉFÉRENCE}

VÉRONIQUE CASTELLOTTI, DIDIER DE ROBILLARD (ed.), France, pays de contact de langues, Louvainla-Neuve, Peeters (Cahiers de l'institut de linguistique de Louvain 28.3-4; 29.1-2), 2003, pp. $153+242$.

1 Cette parution des Cahiers de l'institut de linguistique de Louvain réunit les actes d'un colloque sur les multiples manifestations des contacts de langues dans un pays comme la France, où le brassage interethnique a commencé très tôt. Le tome I s'ouvre avec l'introduction de l'un des éditeurs; les articles sont ensuite regroupés autour de sections thématiques.

2 Didier DE ROBILLARD, Un colloque et ensuite? (13-19) L'un des éditeurs des actes du colloque intervient ici pour montrer les points de convergence entre les différentes contributions qui sont rassemblées dans ces volumes: il s'agit essentiellement du partage d'une linguistique de terrain, à l'approche «compréhensive», épistémologiquement empirique ou «molle», avec une attention marquée pour les retombées sociales, pédagogiques, politiques des contacts entre variétés de langues et/ ou entre langues tout court.

3 La première section, «perspective générale» réunit des études portant sur les approches, les catégories et les problèmes heuristiques de l'enquête de terrain appliquée à l'étude des contacts de langues.

4 Lorenza MONDADA, Pour une approche interactionnelle de la catégorisation des ressources linguistiques par les locuteurs (23-35) S'inspirant de l'analyse conversationnelle d'origine ethnométhodologique, l'A. s'attache à décrire les procédés et les étiquettes de 
catégorisation des ressources linguistiques des locuteurs au cours des échanges linguistiques et aux retombées de celles-ci sur l'activité langagière, sur l'accommodation des stratégies et des choix lexicaux à l'interlocuteur. Les exemples analysés concernent la stigmatisation des gros mots, la réparation d'une fausse prononciation, l'alternance de langues dans un même échange suivant l'interlocuteur sélectionné.

Josiane BOUTET, Christine DEPREZ, Ici et là-bas, public et privé: des catégories à interroger (p. 37-46) La notion de «répertoire verbal», centrale dans les travaux sur les contacts de langues, est ici revue dans une perspective dynamique, basée sur la recatégorisation continuelle qu'opèrent les locuteurs. Cette dynamique est présentée suivant deux axes: l'axe spatial des appartenances linguistiques et culturelles (ici et là-bas), qui révèle souvent l'usage de variétés différentes (une variété rurale pour «là-bas» et une variété urbaine pour «ici»); et l'axe diaphasique (privé - public), analysé dans le cas des relations de service, où la dynamique des répertoires introduit une re-catégorisation de la situation pragmatique. Robert NICOLAÏ, Juliette BOURLIER-BERKOWICZ, A propos d'une conversation de café: remarques sur ce que le corpus peut dire (47-56). Un corpus réalisé avec des adolescentes de la deuxième génération et les commentaires sur les particularités de production qu'il permet de relever sont l'occasion pour une réflexion sur les opérations de construction du corpus préalables à son exploitation. C'est le «paradoxe de l'archéologue», qui détruit le site sur lequel il travaille afin de récupérer les témoignages de l'antiquité: de même, le linguiste doit tenir compte de cette discrimination initiale, où il sélectionne certaines données et en occulte d'autres, et l'expliciter, afin de mieux objectiver son travail.

Les articles de la deuxième section, «Contacts de langues et migrations» focalisent le cas des immigrés des différentes générations ainsi que leur rapport à la langue et à la culture des pays d'origine et d'accueil.

7 Jacqueline BILLIEZ, Stéphanie costa-GAlLIGANI, Vincent LUCCI, Monica MASPÉRI, Agnès MILLET, Cyril TRIMAILLE, Représentations sociales, pratiques langagières et questions identitaires chez des sujets plurilingues (59-78) La problématique du plurilinguisme est abordée dans cette étude à partir de trois groupes sociaux fort différents: des immigrés grenoblois implantés depuis longtemps, des chercheurs migrants temporaires (CMT) dans les secteurs de pointe et de jeunes sourds (bilinguisme LSF-français). Les A. analysent à la fois leurs représentations du bilinguisme et la pratique réelle des deux langues: sentiments à l'égard de la langue d'origine, insécurité linguistique (plus marquée pour les CMT, obligés à jongler avec leur langue, le français et l'anglais fonctionnel) et culturelle (appartenance à deux cultures différentes; exil identitaire et construction d'une identité dans le cas des jeunes, qu'ils soient immigrés ou sourds). Les productions orales montrent une restructuration des répertoires: la communication entre immigrés italiens patoisants de régions différentes et faiblement scolarisés se fait tout de même en italien, mais ce type d'échanges est circonscrit à certains domaines, et c'est le français qui prime dans les contextes formels (le travail, par exemple). L'analyse des pratiques écrites ne montre pas beaucoup le recours à la langue d'origine: celle-ci est réservée à la fonction phatique de garder le contact avec les attaches du pays d'origine (cartes de vœux, lettres etc.), alors que les écrits à fonction informative ou professionnelle sont rédigés en français. (p. 79-91). L'Université d'Amiens développe un projet d'observation des pratiques 
linguistiques en pays picard: celui-ci se caractérise par un patois d'oïl, donc très proche du français, dont la pratique est très vivante, de sorte que les immigrés dans cette région sont généralement confrontés à un plurilinguisme ambiant qui les oblige à se positionner à la fois par rapport au français et au patois. L'A. décrit la démarche adoptée, qui porte sur les parcours individuels d'immigrés d'origines diverses, ainsi que quelques résultats d'enquêtes précédentes, corrélant compréhension d'énoncés picards et vécu linguistique des informateurs.

9 Nicole Gueunier, René kochmann, Des Iraniens à Tours (93-103). Les A. interrogent un corpus d'entretiens d'Iraniens installés en France depuis une vingtaine d'années afin de tracer leurs parcours linguistiques, leurs rapports actuels entre les deux langues qu'ils pratiquent (fréquence et situations d'emploi du persan) ainsi que les représentations qu'ils s'en font avec, à l'horizon, la dimension communautaire que l'exiguïté du nombre de ces immigrés en France ne rend pas très pertinente.

10 Cécile VAN DEN AVENNE, Un récit de ruse. Observations à propos de la question de l'insécurité linguistique (105-113). C'est à travers l'analyse des modalisations autonymiques d'un immigré malien que l'A. essaie d'appréhender les manifestations d'insécurité linguistique typiques des situations de contact. En particulier, elle décrit les situations où le locuteur linguistiquement faible met en œuvre des stratégies pragmatiques (la «ruse») lui permettant d'assumer une position de supériorité vis-à-vis d'un interlocuteur ayant une meilleure maîtrise du français que lui.

11 La section qui ouvre le tome II, «Contacts de langues et cadres institutionnels» examine le problème des langues régionales et de leur enseignement à l'école, en France et dans les DOM, ainsi que le rapport entre une langue non territoriale (le tsigane) et le cadre scolaire.

12 Marielle RISPAIL, Les langues dites régionales: représentations contradictoires des enseignants? (7-19) L'A. se penche sur l'enseignement des langues régionales et tout particulièrement sur les acteurs de cette activité: les enseignants qu'elle a interviewés pour tracer leurs parcours d'appropriation de la langue régionale, les représentations et les rapprochements qu'ils évoquent et les options qu'ils préconisent pour ce type d'enseignement linguistique. C'est surtout à ce niveau que se manifestent les différences les plus nettes entre une approche «spontanéiste» et une approche qui souhaite une prise en charge totale par l'état, dessinant ainsi deux profils-type d'enseignant qui exigent un approfondissement de la notion de contact de langue et qu'on y associe le contact des cultures portées par celles-ci.

13 Thierry Bulot, Claude Caïtucoli, Fabienne leconte, Clara mortamet, Elèves et étudiants dans l'Académie de Rouen: répertoires, pratiques, représentations (21-36). Une enquête sur «l'hétérogénéité linguistique des élèves et des étudiants dans l'académie de Rouen» permet aux A. de revoir un certain nombre d'idées reçues: par exemple le fait que les immigrés manifestent davantage d'hétérogénéité que les autochtones, alors qu'on constate une tendance à un code relativement homogène chez les premiers et une différenciation géographique chez les derniers. Un autre volet de l'enquête a focalisé le problème de la norme enseignée/attendue dans l'enseignement (surnorme académique) et la typologie des écarts des apprenants, ce qui a révélé non seulement des différences de compétence, mais également des différences d'attitudes et d'exigences chez les uns et les autres.

14 Thierry TRÉFAULT, Langues tsiganes: quelques aspects du bilinguisme des familles manouches de Haute Vienne et des enfants scolarisés (37-46). Le problème (socio)linguistique que posent 
les langues tsiganes est avant tout celui du rapport avec le français: il s'agit en effet d'une langue étrangère mais ces groupes sont présents depuis longtemps en France et ne peuvent donc être considérés comme immigrants. En outre le bilinguisme a généralement une fonction hermétique permettant aux manouches de réaffirmer leur identité et leur altérité vis-à-vis des Français. L'un des aspects les plus étonnants de ce bilinguisme concerne les enfants scolarisés qui manifestent de gros problèmes à l'écrit, car la permanence de l'écrit est un aspect étranger à cette culture essentiellement orale.

Marie-Christine HAZAËL-MASSIEUX, Créoles et français dans les DOM: contacts de langues ou conflits? (47-69). L'A. trace un panorama assez complet des créoles dans les DOM, leur situation géographique et les langues en présence dans chaque département. Elle passe ensuite à l'analyse des problèmes liés à l'usage et à la promotion du créole dans ces contextes: le problème de la forme écrite, loin d'être stabilisée et pourtant nécessaire au développement de l'enseignement et de la littérature; le problème de l'enseignement et de la formation des enseignants; l'utilisation du créole dans l'administration et la justice, avec la traduction des textes de loi par exemple; l'utilisation du français dans les médias (presse écrite, radios et télévision, Internet), avec la francisation progressive du créole dans les médias audio.

Robert CHAUDENSON, Les créoles français des DOM sont-ils des langues régionales? (71-87). Après avoir parcouru l'histoire de l'imposition jacobine du français au dépens des dialectes, ainsi que les différentes dispositions de loi depuis la loi Deixonne (1951), L'A. retrace l'histoire parallèle de la diffusion du français dans les DOM et du rôle de la politique et de l'institution scolaire dans cette diffusion. Il remet en question notamment l'absence des créoles de la liste des langues régionales dont l'enseignement est assuré par les circulaires ministérielles, rappelant notamment que le nombre de créolophones actifs égale le nombre des locuteurs de toutes les autres langues régionales réunis et s'interroge sur la pertinence de la définition du créole comme langue régionale.

La section «Villes, contacts de langues», dont la dimension de sociolinguistique urbaine est évidente dès le titre, analyse les codes et les procédés linguistiques à l'œuvre dans des communautés déterminées (jeunes, lycéens, banlieues) et leur corrélés sociaux (ségrégation, valorisation) et culturels (formes d'expression, tel le rap).

Claudine BAvoux, Les parlers jeunes comme indice d'une évolution de la diglossie réunionnaise (91-107). Après avoir brossé un historique de l'implantation du français à la Réunion, l'A. décrit l'émergence récente d'un registre juvénile du français, spécialement lié à la population lycéenne de l'île. Au terme de cet exposé, l'A. s'interroge sur les conséquences de ce type de variation sur la représentation traditionnelle (simpliste ?) de la diglossie créole-français, qui doit évoluer vers une représentation multidimensionnelle.

Michelle auzanneau, margaret Bento, Vincent fayolle, Patricia lambert, Cyril trimaille, Léa AMAR, Angela FERNANDES, Le rap en France et ailleurs: intérêt d'une démarche pluridisciplinaire (109-130). Les A. s'intéressent à la chanson rap francophone (française et gabonaise) comme objet sociolinguistique, permettant à la fois d'observer les variétés «jeunes» en usage, le plurilinguisme légitimé et les représentations des groupes sociaux qui s'affrontent (we/they code), dont la langue devient la représentation et le signal de reconnaissance le plus explicite. 
20 Nathalie AUGER, Véronique FILLOL, Juan LOPEZ, Claudine MOÏSE, L'étude de la violence verbale: enjeux, méthodes, éthique (131-149). S'appuyant sur un corpus d'interactions enregistrées, les A. réfléchissent à la dimension verbale de la violence telle qu'on peut l'observer dans des classes de quartiers dits «difficiles» où s'affrontent des locuteurs pourvus de compétences et de légitimités différentes (les enseignants et les élèves), le plus souvent enfermés dans des représentations stéréotypées. La présence physique de l'enquêteur soulève de nombreuses questions quant à son influence sur les résultats de l'observation elle-même, ce que les A. ne manquent pas de souligner.

21 Karine GIACOMI, Nathalie BINISTI, Christelle ASSEF, Médéric GASQUET-CYRUS, Les voix de Marseille (151-164). Les A. ont mené une enquête sur les variétés en contact et sur les représentation sociolinguistiques des locuteurs du quartier de la Plaine à Marseille et montrent comment la coexistence de plusieurs parlures (occitan, français régional, langage des cités) recouvre des différences sociolinguistiques importantes et contribue a renforcer l'identité de groupes ou de réseaux sociolinguistiques via la territorialisation spatiale des parlures («français des poissonnières du Vieux Port», «français des quartiers Nord» «accent rue Paradis», «accent de Mazargues» etc.).

22 Frank JABLONKA, Sociolinguistique suburbaine: quelle langue a droit de cité en France? (165-177). Une enquête sur «normes spatiales et identités sociales» menée dans une cité à Besançon est l'occasion d'une réflexion sur la légitimité (le «droit de cité») octroyée à la variété sous-standard qui est dominante dans ces espaces, sur les rapports que celleci entretient avec la langue officielle et sur la variation à son intérieur, suite aux contacts de langues et aux structures de groupe, intrinsèquement dissipatives qui président à l'élaboration de ces codes linguistiques identitaires.

23 La dernière section, «Français, variation, contacts, représentations» réunit des études portant essentiellement sur les structures linguistiques et sur les variations que cellesci subissent du fait du contact de locuteurs aux normes différentes. C'est le cas notamment des articles sur les indéfinis et les formes interrogatives, tandis que les deux articles suivants s'intéressent à la variation diamésique (le français sur internet) et à la représentation spontanée que les locuteurs se font de la variation.

24 Paul CAPPEAU, Des sujets indéfinis (181-191). Partant du constat du flou caractérisant la classe des indéfinis, l'A. s'intéresse en particulier à deux indéfinis en position sujet: certains et il y en a qui, dont il étudie les distributions en français parlé. Ce sont des paramètres internes (certains non anaphorique comporte le trait /+humain/ et apparaît avec des verbes de parole, tandis que certains anaphorique n'a pas de restrictions sémantiques et apparaît avec des verbes au passif ou intransitifs) et externes (les débats politiques présentent un grand nombre de certains non anaphorique, alors que les conversations informelles manifestent davantage la présence de l'anaphorique) qui président à la répartition des usages. Les paramètres internes sont largement comparables pour il y en a qui, tandis que les paramètres externes excluent cette forme des situations plus formelles.

Virginie QUILLARD, Quelle variation pour l'interrogation? Quelques éléments de réponse à travers le modèle par inversion (193-200). Après avoir illustré la différence entre la variation phonétique et grammaticale et avoir postulé la présence d'invariants grammaticaux à condition de délimiter des contextes très ciblés, l'A. analyse le champ de l'interrogation par inversion, dont elle identifie deux contextes de variation: un contexte formel (où se manifeste une variation diastratique) et un contexte 
pragmatique, où l'inversion est utilisée pour des questions rhétoriques ou ironiques. Dans ce cas, la composante diastratique n'est plus pertinente.

Isabelle PIEROZAK, Eléments de réflexion vers un cadre théorique pour l'étude du français sur Internet: variantes en contact et emprunts à la créolistique (201-222). Les pratiques scripturales caractérisant la communication via l'Internet configurent un espace de variation difficile à décrire uniquement à partir des données internes, mais réclament la prise en compte des données externes articulant les communautés virtuelles auxquelles s'identifient les locuteurs/scripteurs. C'est justement en raison de cette articulation que l'A. utilise certains acquis de la créolistique pour expliquer la dynamique de cette variation tout en démontrant que celle-ci ne peut pas être considérée comme un créole.

Véronique CASTELLOTTI, Didier DE ROBILLARD, Des français devant la variation: quelques hypothèses (223-240). A partir d'une enquête sociolinguistique recueillant les réactions de locuteurs français face à des enregistrements de variantes de la langue nationale, les A. réfléchissent sur le concept même de variation, sur l'existence (certes contradictoire) de variantes prototypiques et sur les groupes sociaux «autorisés», qui ne peuvent pas porter de traces de parlure locale, parmi lesquels les professionnels des médias jouent actuellement un rôle central. 